\title{
Analyse texturale et géochimique d'un polissoir à rainures du gisement magdalénien de Duruthy (Sorde, Landes, France)
}

Morgane Dachary, Catherine Deniel, Frédéric Plassard, Pierre Boivin et Jean-Luc Devidal

\section{(2) OpenEdition}

Journals

Édition électronique

URL : http://journals.openedition.org/paleo/2414

DOI : $10.4000 /$ paleo.2414

ISSN : 2101-0420

Éditeur

SAMRA

\section{Édition imprimée}

Date de publication : 15 décembre 2012

Pagination : 315-322

ISSN : 1145-3370

\section{Référence électronique}

Morgane Dachary, Catherine Deniel, Frédéric Plassard, Pierre Boivin et Jean-Luc Devidal, « Analyse texturale et géochimique d'un polissoir à rainures du gisement magdalénien de Duruthy (Sorde, Landes, France) », PALEO [En ligne], 23 | 2012, mis en ligne le 29 avril 2013, consulté le 24 juillet 2020. URL : http://journals.openedition.org/paleo/2414 ; DOI : https://doi.org/10.4000/paleo.2414

Ce document a été généré automatiquement le 24 juillet 2020.

\section{cc)}

PALEO est mis à disposition selon les termes de la licence Creative Commons Attribution - Pas d'Utilisation Commerciale - Pas de Modification 4.0 International. 


\title{
Analyse texturale et géochimique d'un polissoir à rainures du gisement magdalénien de Duruthy (Sorde, Landes, France)
}

\author{
Morgane Dachary, Catherine Deniel, Frédéric Plassard, Pierre Boivin et \\ Jean-Luc Devidal
}

\begin{abstract}
Dans la littérature sur le Magdalénien, il n'est pas rare de rencontrer la mention de polissoirs à rainures. Ces objets, creusés de sillons plus ou moins profonds, ont très tôt attiré l'attention des fouilleurs qui les ont interprétés comme des polissoirs liés au travail de l'os ou du bois de cervidé. Citons, par exemple, le gisement de La Madeleine (Capitan et Peyrony 1928), ou celui des Combarelles (Rivière 1894). En général, ces polissoirs sont en grès plus ou moins tendre. Cependant, d'autres matières ont parfois été utilisées : c'est le cas de la pierre ponce. La possible découverte d'un exemplaire de ce type dans le gisement de Duruthy (Sorde, Landes) a conduit à réaliser une analyse texturale et géochimique du matériau. Sa nature ponceuse a été confirmée, permettant d'avancer une hypothèse quant à sa provenance. Parallèlement, un inventaire des pièces comparables a été dressé sur la base d'un dépouillement bibliographique. Cette recherche co-financée par le Ministère de la Culture et le Conseil Général des Landes, fait ici l'objet d'un bilan d'étape alors que notre projet se poursuit.
\end{abstract}

\section{1- Inventaire des polissoirs à rainures en pierre ponce}

2 Le polissoir à rainures en pierre ponce le plus anciennement mentionné dans la littérature est celui de Gourdan, signalé par E. Piette dès 1873 : "J'ai trouvé dans la caverne une pierre ponce qui leur avait servi à polir des aiguilles et des poinçons. Cette pierre paraît provenir des terrains volcaniques d'Agde. " (Piette 1873 - p. 413). Plus tard, E. Passemard signale également des polissoirs à Isturitz : "Enfin, il y a des fragments de pierre ponce utilisés pour l'affûtage des pointes osseuses » (Passemard 1944 - p. 45 et Pl. XX). 
Un autre exemplaire est décrit sur le versant espagnol dans le gisement d'Aitzbitarte IV (fig. 1) par J.-M. de Barandiaran (1965) qui en donne un dessin où l'on repère une perforation en plus des rainures. Enfin, le gisement de Bourrouilla à Arancou fournit une pièce comparable, quoique non perforée. Découverte dans les déblais de la fouille clandestine (fig. 2), elle fut publiée par F.-X. Chauvière (Chauvière in Chauchat et al. 1999).

3 En l'état actuel de nos recherches, le corpus des polissoirs à rainures en pierre ponce est donc limité (tabl. 1) puisque seulement cinq sites sont concernés et, mis à part Isturitz, chacun n'a fourni qu'un seul exemplaire. Le cas d'Isturitz semble donc original, mais il nous faudra examiner le matériel pour valider la mention très sommaire de ces pièces par E. Passemard. Notons que tous les sites inventoriés sont concentrés dans la moitié occidentale des Pyrénées.

4 Enfin, les données chronostratigraphiques associées à ces objets sont très limitées. Aucun ne provient de fouilles récentes apportant une lecture fine de la stratigraphie. Cependant, tous ont été découverts dans des sites ayant livré des niveaux magdaléniens moyens et/ou supérieurs, ce qui peut suggérer que le matériau ponceux utilisé pour fabriquer ces divers objets pourrait avoir la même origine.

Figure 1 - Aitzbitarte IV. Polissoir à rainures en pierre ponce. (d'après Barandiaran 1965).

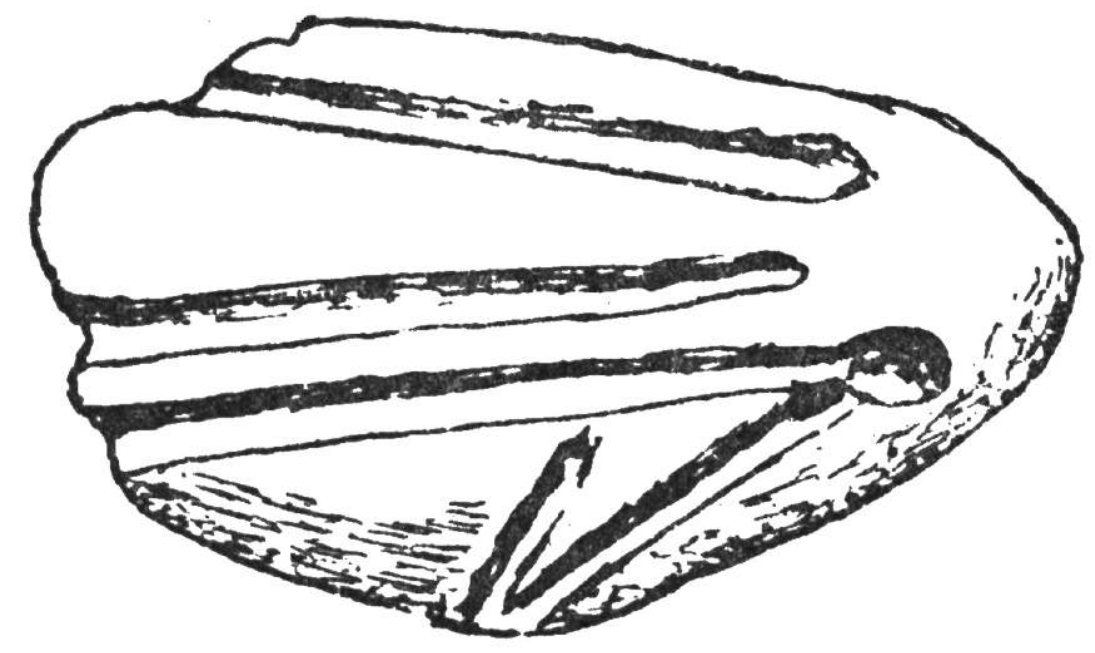

O $3 \mathrm{~cm}$ 
Figure 2 - Arancou. Polissoir à rainures en pierre ponce. (Dessin F.-X. Chauvière et photo F. Plassard).

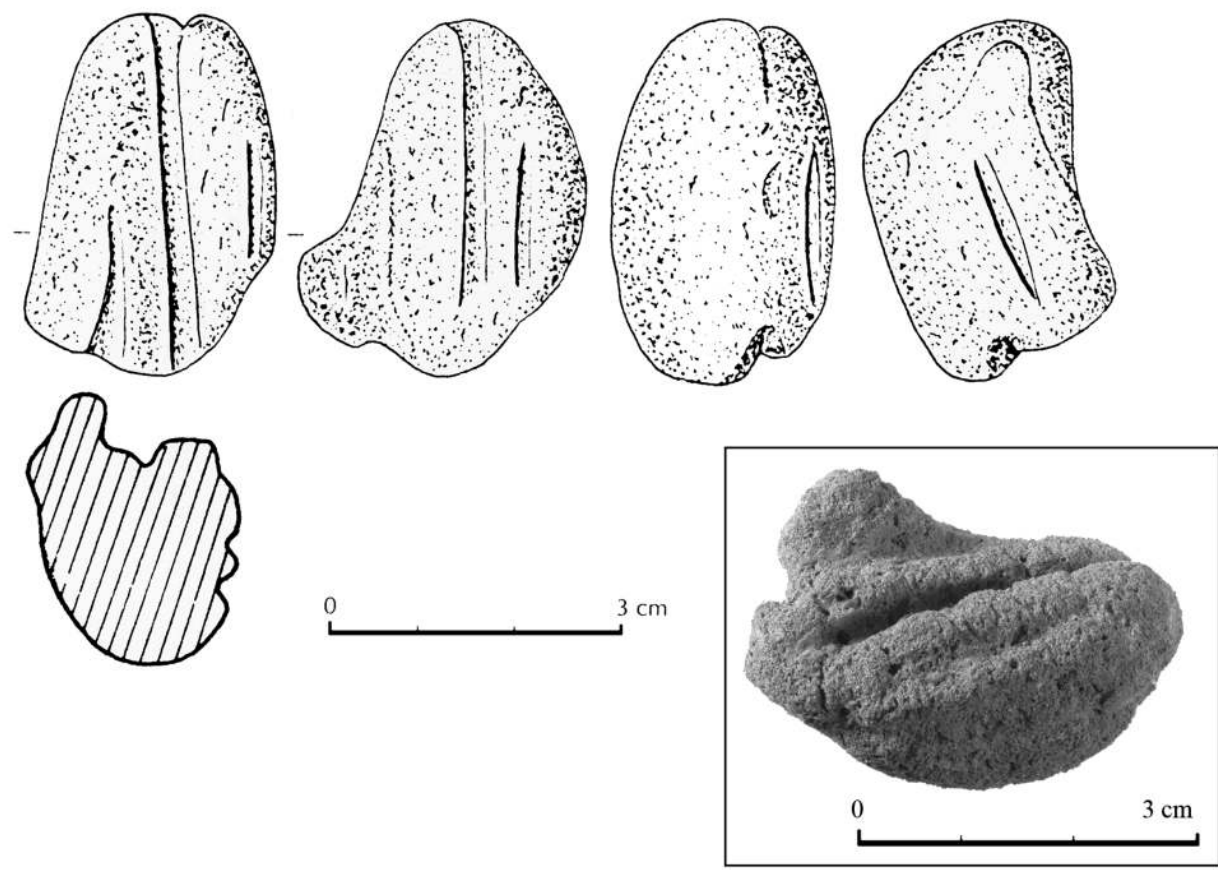

Tableau 1 - Inventaire des sites ayant livré des polissoirs à rainures en pierre ponce.

\begin{tabular}{l|l|l|l} 
Site & Stratigraphie & Description & Références bibliographiques \\
\hline \hline Aitzbitarte IV & $\begin{array}{l}\text { Couche IV "tierra } \\
\text { oscurra" }\end{array}$ & $\begin{array}{l}\text { Polissoir à rainures avec trou de } \\
\text { suspension }\end{array}$ & Barandiaran (de) 1965 \\
\hline Arancou & $\begin{array}{l}\text { Fouille } \\
\text { clandestine }\end{array}$ & Polissoir à rainures & $\begin{array}{l}\text { Chauvière in Chauchat } \\
\text { et al. 1999 }\end{array}$ \\
\hline Duruthy & Remanié & $\begin{array}{l}\text { Polissoir à rainures avec trou de } \\
\text { suspension }\end{array}$ & Inédit \\
\hline Gourdan & $\begin{array}{l}\text { "assises } \\
\text { représentant } \\
\text { l'Age du renne" }\end{array}$ & $\begin{array}{l}\text { "une pierre ponce qui leur avait } \\
\text { servi à polir des aiguilles et des } \\
\text { poinçons" }\end{array}$ & Piette 1873 \\
\hline Isturitz & $\begin{array}{l}\text { Couche E } \\
\text { "des fragments de pierre ponce } \\
\text { utilisés pour l'affütage des pointes } \\
\text { osseuses" }\end{array}$ & Passemard 1944
\end{tabular}

\section{2 - Le polissoir de Duruthy}

5 Le réexamen du matériel de l'abri Duruthy par l'un de nous (MD) a permis l'identification de plusieurs polissoirs à rainures (fig. 3). Ceux-ci avaient été considérés initialement comme étant tous en grès (Arambourou 1978). Ce matériau, assez tendre, parfois très altéré est peu dense. Néanmoins, l'un de ces polissoirs a un aspect bien différent des autres (couleur plus grise, paillette vitreuse, porosité importante) et une densité plus faible encore, qui accrédite l'hypothèse de la ponce (fig. 4). En outre, il possède une perforation biconique évoquant un trou de suspension comme celui décrit à Aitzbitarte IV. 
6 Malheureusement, ce polissoir de Duruthy, issu des fouilles conduites par R. Arambourou entre 1959 et 1984, a été découvert dans des sédiments remaniés. Aucune attribution stratigraphique n'est donc possible. On peut cependant noter que parmi les autres polissoirs à rainures (en grès) découverts dans ce gisement, ceux pour lesquels on dispose d'informations stratigraphiques appartiennent aux couches 3 et 4 , respectivement attribuées au Magdalénien supérieur et moyen.

7 Pour déterminer la nature de ce matériau et entreprendre une recherche sur son origine, une analyse texturale et géochimique a donc été conduite au Laboratoire Magmas et Volcans (UMR 6524 CNRS) de Clermont-Ferrand.

Figure 3 - Duruthy. Polissoir en grès. (๑ F. Plassard).

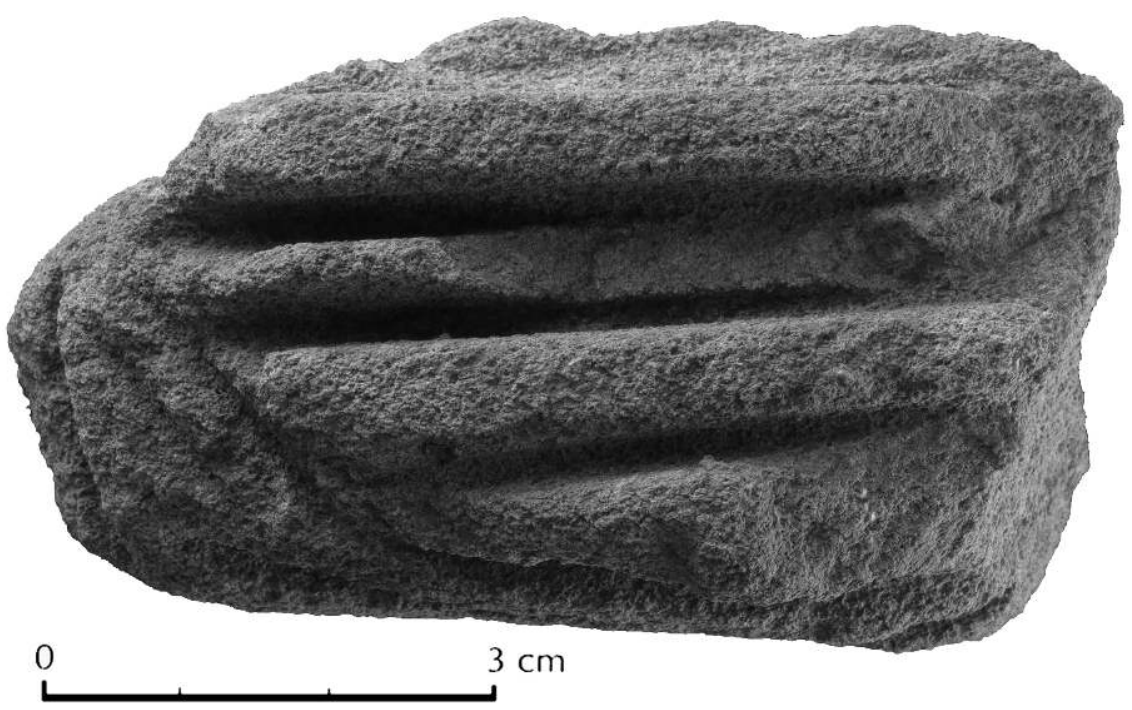

\section{1 - Texture et pétrologie du polissoir de Duruthy}

\subsection{1 - Résultats de l'analyse macroscopique et binoculaire de l'échantillon}

Même si une porosité similaire à celle de ce polissoir peut s'observer pour certaines roches sédimentaires altérées par des processus secondaires, mettant en jeu des dissolutions préférentielles, une densité aussi faible que celle de cet échantillon n'en est pas une caractéristique habituelle. Une faible densité est en effet plus typique de certaines roches volcaniques appelées ponces, avec des compositions bien particulières.

9 Nous avons profité de la présence d'un petit fragment détaché naturellement du polissoir pour pousser plus loin nos investigations. Une analyse binoculaire de celui-ci a été effectuée. Les deux photos (fig. 5) permettent d'observer la porosité importante de la roche constituant le polissoir de Duruthy. De plus, les zones transparentes apparaissant sur ces photos évoquent du verre qui semble constituer les parois des vésicules. Ces deux observations confortent l'hypothèse que le matériel ayant servi à la fabrication de ce polissoir n'est probablement pas une roche sédimentaire mais, plus vraisemblablement, une roche volcanique, ponceuse. 


\subsection{2 - Microtextures du polissoir de Duruthy par microscopie électronique à} balayage (MEB) utilisé en mode imagerie

10 L'utilisation du microscope électronique à balayage, en mode imagerie, permet d'observer les microtextures des échantillons.

11 Sur la figure 6a, on voit, avec beaucoup plus de détails, les parois des vésicules précédemment observées à la binoculaire. La figure $6 \mathrm{~b}$ est un zoom de ces parois de vésicule. On y voit nettement des figures de dissolution, ainsi que des petits cristaux en baguettes.

12 La figure $6 \mathrm{c}$ montre que ces baguettes sont associées au matériel des parois et font donc partie intégrante de la roche analysée. En effet, ces baguettes sont recoupées par les figures de dissolution observées sur les parois.

\section{2 - Composition géochimique du polissoir}

\subsection{1 - Détermination qualitative de la composition géochimique par microscopie électronique à balayage (MEB) en dispersion d'énergie (EDS)}

13 Pour vérifier qu'il pouvait s'agir d'un matériel d'origine volcanique, et non d'un matériel sédimentaire (grès à structure poreuse), nous avons d'abord effectué une détermination qualitative, mais non quantitative, de la composition chimique de l'échantillon. Cette approche qualitative a été préférée, dans un premier temps, parce qu'elle est totalement non destructive.

14 La paroi des vésicules visibles sur les photos MEB a donc été analysée avec un microscope électronique à balayage disposant d'un système de microanalyse chimique, fonctionnant en dispersion d'énergie (EDS).

Le résultat de l'analyse a révélé une composition de verre siliceux pour ces parois de vésicules, confirmant ainsi qu'il s'agissait bien d'un matériel d'origine volcanique, vraisemblablement rhyolitique.

Figure 4 - Duruthy. Polissoir à rainures en pierre ponce. (๑ F. Plassard).

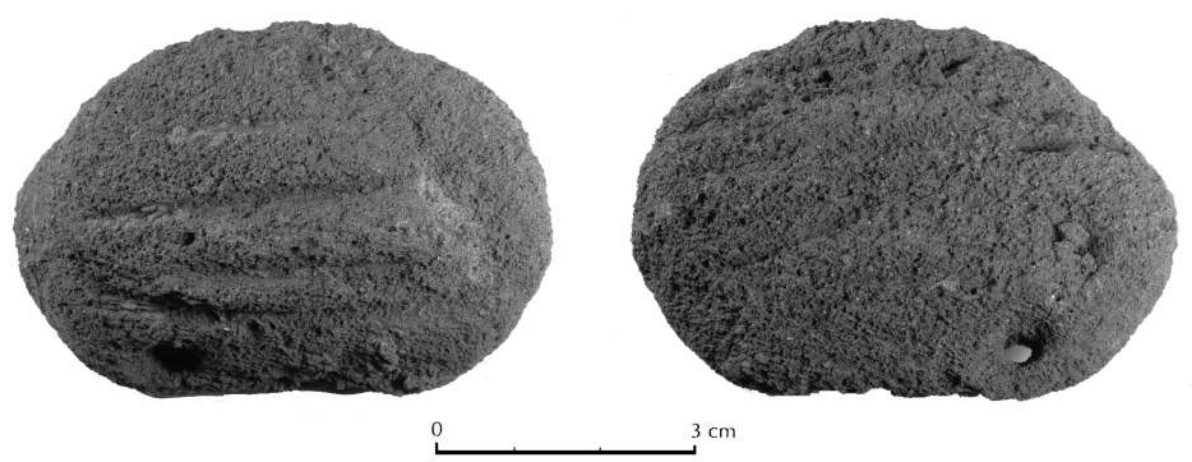


Figure 5 - Duruthy. Fragment du polissoir observé à la loupe binoculaire. (@ P. Boivin).

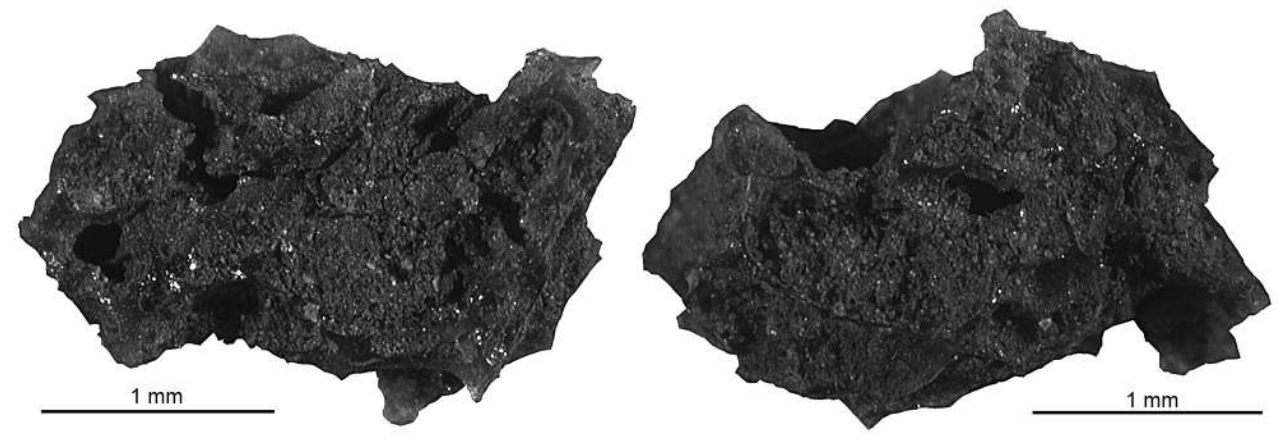

Figure 6 - Duruthy. Fragment du polissoir observé au MEB. (๑ laboratoire MEB).

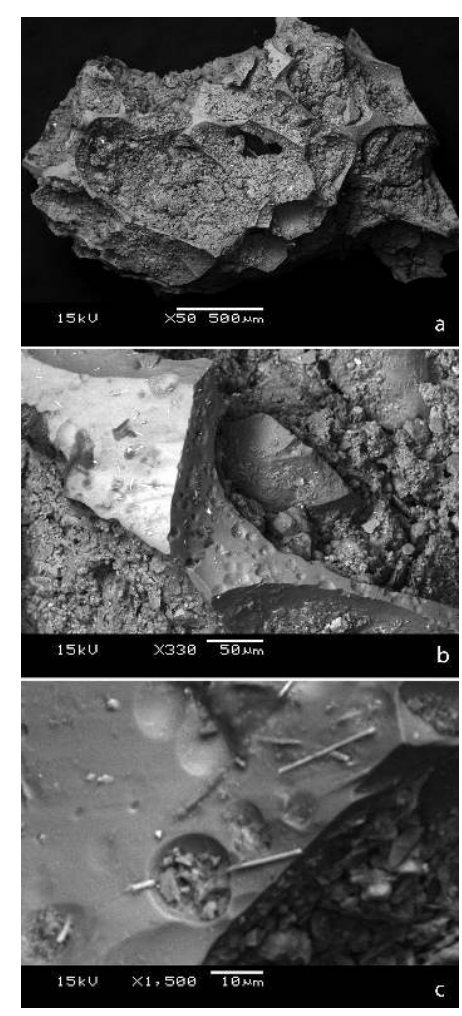

\subsection{2 - Détermination précise de la composition géochimique par microsonde électronique}

16 Ayant ensuite obtenu l'autorisation de travailler librement sur le fragment millimétrique détaché du polissoir, nous avons pu envisager une analyse plus précise de sa composition géochimique. Ce fragment a été inclus dans de la résine puis abrasé et poli avant de procéder à des mesures, quantitatives cette fois, à la microsonde électronique (WDS Cameca SX100 de l'UMR 6524 CNRS).

17 Ces mesures ont été effectuées en utilisant une tension d'accélération de $15 \mathrm{kV}$. Une faible intensité de courant ( $8 \mathrm{nA}$ voire $4 \mathrm{nA}$ ) et un faisceau défocalisé (diamètre de $20 \mu \mathrm{m}$ ) ont été utilisés afin de réduire au maximum les pertes de Na, inhérentes à ce type de mesures. Huit analyses ponctuelles ont ainsi été effectuées sur les parois des vésicules et une analyse sur les cristaux en baguettes associés et décrits section 2.1.2. 
Parallèlement, le standard international A99 (verre basaltique du Kilauea, Hawaii, Jarosewich et al. 1979; Thornber et al. 2002) a été mesuré sept fois afin de fournir un contrôle externe de l'exactitude et de la reproductibilité de ces mesures.

18 Les résultats individuels (tabl. 2) sont très cohérents entre eux (cf. écarts-type sur les moyennes). De plus, les valeurs obtenues sur le standard sont indistinguables (dans la limite des écarts-type) de celles de la littérature et de celles antérieurement obtenues à l'UMR 6524 sur le même instrument (Oladottir et al. 2011), soulignant la fiabilité de ces résultats. Même le $\mathrm{Na} 2 \mathrm{O}$, susceptible d'être entaché d'une erreur supérieure aux autres oxydes à cause de sa volatilité lors des mesures (cf. écart-type un peu supérieur), est ainsi déterminé avec une bonne précision, comme l'indiquent les résultats sur le standard A99. Ces résultats très précis obtenus à la microsonde confirment que le matériel constituant les parois des vésicules est bien du verre rhyolitique.

Par ailleurs, la composition des petits cristaux en baguettes, et notamment les pourcentages en Fe et $\mathrm{Mg}$, sont tout à fait comparables à ceux que l'on observe dans les pyroxènes des roches volcaniques différenciées. Compte tenu de la nature de la roche, de cet enrichissement en Fe et $\mathrm{Mg}$ et de l'habitus de ces cristaux, ces baguettes sont vraisemblablement de petits cristaux de pyroxène.

Tableau 2 - Duruthy. Analyses à la microsonde électronique des parois des vésicules du polissoir et des cristaux en baguettes (pyroxène) sur ces parois. $\mathrm{n}$ correspond au nombre d'analyses utilisées pour les moyennes. * Analyse de moins bonne qualité que pour la paroi des vésicules à cause de la petite taille des cristaux.

\begin{tabular}{|c|c|c|c|c|c|c|c|c|c|c|c|c|}
\hline \multicolumn{2}{|c|}{ Standard international A99 } & \multicolumn{10}{|c|}{ Oxydes \% (poids) } & \\
\hline \multicolumn{2}{|c|}{$\mathrm{n}^{\circ}$ d'analyse } & $\mathrm{SiO}_{2}$ & $\mathrm{TiO}_{2}$ & $\mathrm{Al}_{2} \mathrm{O}_{3}$ & $\mathrm{FeO}$ & $\mathrm{MnO}$ & $\mathrm{MgO}$ & $\mathrm{CaO}$ & $\mathrm{Na}_{2} \mathrm{O}$ & $\mathrm{K}_{2} \mathrm{O}$ & $\mathrm{P}_{2} \mathrm{O}_{5}$ & Total \\
\hline \multicolumn{2}{|l|}{1} & 51,34 & 4,13 & 12,65 & 13,11 & 0,28 & 5,08 & 9,02 & 2,64 & 0,85 & 0,50 & 99,61 \\
\hline \multicolumn{2}{|l|}{2} & 51,37 & 4,08 & 12,84 & 13,50 & 0,20 & 5,04 & 9,20 & 2,77 & 0,84 & 0,44 & 100,27 \\
\hline \multicolumn{2}{|l|}{3} & 50,78 & 4,01 & 12,82 & 12,95 & 0,20 & 5,00 & 9,10 & 2,68 & 0,88 & 0,48 & 98,91 \\
\hline \multicolumn{2}{|l|}{4} & 50,49 & 4,18 & 13,04 & 13,17 & 0,28 & 5,07 & 8,94 & 2,77 & 0,84 & 0,49 & 99,27 \\
\hline \multicolumn{2}{|l|}{5} & 51,24 & 4,15 & 12,60 & 13,67 & 0,23 & 5,04 & 9,26 & 2,68 & 0,83 & 0,53 & 100,24 \\
\hline \multicolumn{2}{|l|}{6} & 51,22 & 4,18 & 12,54 & 13,54 & 0,25 & 5,05 & 9,05 & 2,80 & 0,89 & 0,48 & 99,99 \\
\hline \multicolumn{2}{|l|}{7} & 51,12 & 4,15 & 12,68 & 13,18 & 0,17 & 5,07 & 9,09 & 2,55 & 0,86 & 0,40 & 99,25 \\
\hline \multirow{2}{*}{ Cette étude } & $\begin{array}{c}\text { moyenne } \\
(\mathrm{n}=7)\end{array}$ & 51,08 & 4,13 & 12,74 & 13,30 & 0,23 & 5,05 & 9,09 & 2,70 & 0,86 & 0,47 & 99,65 \\
\hline & ècart-type & 0,33 & 0,06 & 0,17 & 0,27 & 0,04 & 0,03 & 0,11 & 0,09 & 0,02 & 0,04 & 0,53 \\
\hline \multirow{2}{*}{$\begin{array}{l}\text { Oladottir et al. (2011) } \\
\text { (UMR } 6524 \text { CNRS) }\end{array}$} & $\begin{array}{l}\text { moyenne } \\
(\mathrm{n}=1536)\end{array}$ & 51,00 & 4,17 & 12,65 & 13,20 & 0,20 & 5,01 & 9,14 & 2,69 & 0,86 & 0,46 & 99,35 \\
\hline & écart-type & 0,54 & 0,06 & 0,15 & 0,29 & 0,07 & 0,09 & 0,09 & 0,11 & 0,02 & 0,02 & \\
\hline $\begin{array}{c}\text { Jarosewich et al. } \\
\text { (1979) }\end{array}$ & & 50,94 & 4,06 & 12,49 & 13,30 & 0,15 & 5,08 & 9,30 & 2,66 & 0,82 & 0,38 & 99,18 \\
\hline \multirow{2}{*}{ Thomber et al. (2002) } & $\begin{array}{c}\text { moyenne } \\
(\mathrm{n}=418)\end{array}$ & 51,06 & 3,95 & 12,44 & 13,15 & 0,19 & 5,04 & 9,04 & 2,72 & 0,82 & 0,43 & 98,84 \\
\hline & écart-type & 0,46 & 0,09 & 0,13 & 0,16 & 0,02 & 0,10 & 0,11 & 0,16 & 0,03 & 0,03 & \\
\hline
\end{tabular}

\begin{tabular}{|c|c|c|c|c|c|c|c|c|c|c|c|c|}
\hline \multirow{2}{*}{$\begin{array}{c}\text { Polissoir de Duruthy } \\
\text { Paroi des vésicules } \\
n^{\circ} \text { d'analyse } \\
\end{array}$} & \multicolumn{10}{|c|}{ Oxydes $\%$ (poids) } & & \multirow{2}{*}{$\begin{array}{l}\text { Caractéristiques } \\
\text { faisceau }\end{array}$} \\
\hline & $\mathrm{SiO}_{2}$ & $\mathrm{TiO}_{2}$ & $\mathrm{Al}_{2} \mathrm{O}_{3}$ & $\mathrm{FeO}$ & $\mathrm{MnO}$ & $\mathrm{MgO}$ & $\mathrm{CaO}$ & $\mathrm{Na}_{2} \mathrm{O}$ & $\mathrm{K}_{2} \mathrm{O}$ & $\mathrm{P}_{2} \mathrm{O}_{5}$ & Total & \\
\hline 1 & 75,66 & 0,18 & 13,32 & 1,21 & 0,12 & 0,02 & 0,77 & 4,35 & 3,99 & 0,00 & 99,64 & $8 \mathrm{nA}, 20 \mu \mathrm{m}$ \\
\hline 2 & 74,87 & 0,18 & 13,58 & 1,15 & 0,09 & 0,01 & 0,87 & 4,83 & 3,83 & 0,07 & 99,49 & $4 \mathrm{nA}, 20 \mu \mathrm{m}$ \\
\hline 3 & 75,06 & 0,18 & 13,54 & 1,24 & 0,12 & 0,03 & 0,75 & 4,22 & 4,02 & 0,10 & 99,25 & $4 \mathrm{nA}, 20 \mu \mathrm{m}$ \\
\hline 4 & 75,10 & 0,15 & 13,45 & 1,12 & 0,10 & 0,05 & 0,76 & 4,44 & 4,01 & 0,00 & 99,18 & $8 \mathrm{nA}, 20 \mu \mathrm{m}$ \\
\hline 5 & 74,99 & 0,17 & 13,62 & 1,30 & 0,04 & 0,05 & 0,77 & 4,35 & 4,02 & 0,08 & 99,39 & $8 \mathrm{nA}, 20 \mu \mathrm{m}$ \\
\hline 6 & 74,80 & 0,17 & 13,74 & 0,94 & 0,06 & 0,02 & 0.75 & 3,98 & 4,07 & 0,00 & 98,53 & $4 \mathrm{nA}, 20 \mu \mathrm{m}$ \\
\hline 7 & 74,45 & 0,20 & 13,67 & 1,00 & 0,00 & 0,00 & 0,77 & 3,99 & 4,00 & 0,00 & 98,09 & $4 \mathrm{nA}, 20 \mu \mathrm{m}$ \\
\hline 8 & 75,50 & 0,17 & 13,25 & 1,31 & 0,07 & 0,04 & 0,76 & 4,22 & 4,02 & 0,01 & 99,35 & $8 \mathrm{nA}, 20 \mu \mathrm{m}$ \\
\hline moyenne $(n=8)$ & 75,05 & 0,18 & 13,52 & 1,16 & 0,08 & 0,03 & 0,78 & 4,30 & 4,00 & 0,03 & 99,11 & \\
\hline écart-type & 0,39 & 0,01 & 0,17 & 0,13 & 0,04 & 0,02 & 0,04 & 0,27 & 0,07 & 0,04 & 0,53 & \\
\hline $\begin{array}{l}\text { Polissoir de Duruthy } \\
\text { Cristaux en baguettes }\end{array}$ & \multicolumn{10}{|c|}{ Oxydes \% (poids) } & & \\
\hline $\mathrm{n}^{\circ}$ d'analyse & $\mathrm{SiO}_{2}$ & $\mathrm{TiO}_{2}$ & $\mathrm{Al}_{2} \mathrm{O}_{3}$ & $\mathrm{FeO}$ & $\mathrm{MnO}$ & $\mathrm{MgO}$ & $\mathrm{CaO}$ & $\mathrm{Na}_{2} \mathrm{O}$ & $\mathrm{K}_{2} \mathrm{O}$ & Total & & \\
\hline 1 & 48,88 & 0,48 & 1,83 & 24,21 & 0,81 & 8,26 & 13,31 & 0,33 & 0,09 & $98,20 *$ & & \\
\hline
\end{tabular}




\section{3 - Conclusion sur la nature du matériel géologique constituant le polissoir à rainures du gisement préhistorique de Duruthy} Piette ne peut pas être retenue dans le cas du polissoir de Duruthy puisque aucun des matériaux volcaniques de ce secteur ne correspond à de la véritable ponce. De même, il n'y a pas de correspondance entre la composition géochimique de la ponce du polissoir et celle des ponces du Massif central.

En revanche, la concentration des gisements ayant livré ces objets à proximité du littoral atlantique serait compatible avec un approvisionnement côtier de matériau arrivé par flottation, à la suite de l'éruption d'un volcan sur une île de l'Atlantique ou des Caraïbes (voire sur le continent américain ?). A ce jour, le dépouillement, non encore exhaustif, des données bibliographiques sur des éruptions de la fin du Pléistocène n'a pas fait apparaître d'événement éruptif ayant produit des matériaux dont la signature géochimique est similaire à celle du polissoir de Duruthy. Cette hypothèse, même si elle nous semble la plus vraisemblable, n'est donc, pour l'instant, pas vérifiée. Elle constituerait, néanmoins, une preuve supplémentaire, mais inattendue, de l'exploitation du littoral par les chasseurs-cueilleurs du Tardiglaciaire du sud-ouest de la France et du nord de l'Espagne (Pétillon 2008 ; Corchón-Rodriguez et Àlvarez-Fernandez 2008). Notons toutefois que des phénomènes analogues ont été

PALEO, 23 | 2012 
documentés au Néolithique, dans le nord de l'Europe (Écosse, Scandinavie) ainsi que, de façon plus ponctuelle, en Bretagne (Giot 1972).

En outre, ce " gîte » côtier de pierre ponce aurait été événementiel, et la conservation de ce matériau sur le littoral vraisemblablement de courte durée. Si des analyses sur les autres polissoirs répertoriés amenaient à démontrer l'identité du matériau d'un site à l'autre, cela constituerait un élément significatif pour apprécier la contemporanéité relative des sites.

A ce stade, notre projet sur les polissoirs en pierre ponce n'est donc pas achevé. D'une part, parmi les objets identifiés dans la littérature, seuls ceux d'Arancou et de Duruthy ont pu être étudiés directement. D'autre part, le polissoir de Duruthy est le seul à avoir pu faire l'objet d'analyses géochimiques, et seulement pour les éléments majeurs. Dans l'avenir, la multiplication des analyses géochimiques (éléments majeurs mais aussi en traces, indispensables pour pouvoir identifier la source volcanique du matériel avec une bonne fiabilité) sur d'autres objets, la poursuite des recherches bibliographiques et le dépouillement des données géochimiques existantes sur les évènements volcaniques en Atlantique à la fin du Pléistocène permettront de répondre aux questions encore en suspens.

\section{BIBLIOGRAPHIE}

ARAMBOUROU R. (dir.) 1978 - Le gisement préhistorique de Duruthy à Sorde-l'Abbaye (Landes), Société Préhistorique Française, Mémoire 13, 158 p.

BARANDIARAN J.-M. (de) 1965 - Excavaciones en la caverna de Aitzbitarte IV, Noticiario arqueologico hispanico, tome VII, p. 35-48.

CAPITAN L., PEYRONY D. 1928 - La Madeleine : son gisement, ses industries et ses cuvres d'art, Publications de l'Institut International d'Anthropologie, Paris : Librairie Nourry, 125 p., 70 fig., 19 pl.

CHAUCHAT CI., FONTUGNE M., HATTE CH., DACHARY M., BONNISSENT D., CHAUVIÈRE F.-X., ROUSSOT A., FRITZ C., FOSSE Ph., EASTHAM A., MARTIN H., LE GALL O., GAMBIER D. 1999 L'habitat Magdalénien de la grotte Bourrouilla à Arancou (Pyrénées Atlantiques). Gallia Préhistoire, tome 41, p. 1-151.

CORCHÓN-RODRÍGUEZ M.-S., ÁLVAREZ-FERNÁNDEZ E. 2008 - Nuevas evidencias de restos de mamíferos marinos en el Magdaleniense : los datos de La Cueva de Las Caldas (Asturias, España). Munibe, 59, p. 47-66.

GIOT P.-R. 1972 - La pierre ponce des sites préhistoriques de Bretagne. Bulletin de la Société Préhistorique française, tome $69 \mathrm{n}^{\circ}$ 5, p. 159-160.

JAROSEWICH E., NELEN J.A., BORBERG J.A. 1979 - Electron microprobe reference samples for mineral analysis. In : Fudali R.F. (ed.) Smithsonian Institution Contributions to the Earth Sciences $\mathrm{n}^{\circ}$ 22. Smithsonian Institution Press, p. 68-72. 
OLADOTTIR B.A., SIGMARSSON O., LARSEN G., DEVIDAL J.-L. 2011 - Provenance of basaltic tephra from Vatnajökull subglacial volcanoes, Iceland, as determined by major- and trace-element analyses. The Holocene 21(7), p. 1037-1048.

PASSEMARD E. 1944 - La caverne d'Isturitz en pays basque. La Préhistoire, 9, p. 7-95.

PÉTILLON J.-M. 2008 - First evidence of a whale-bone industry in the western European Upper Paleolithic : Magdalenian artifacts from Isturitz (Pyrénées-Atlantiques, France). Journal of Human Evolution 54, p. 720-726.

PIETTE E. 1873 - Sur la grotte de Gourdan, sur la lacune que plusieurs auteurs placent entre l'âge du renne et celui de la pierre polie, et sur l'art paléolithique dans ses rapports avec l'art gaulois. Bulletin de la Société d'anthropologie de Paris, $\mathrm{II}^{\circ}$ Série, tome 8, p. 384-425.

THORNBER C.R., SHERROD D.R., SIEMS D.R., HELIKER C.C., MEEKER G.P., OSCARSSON R.L. et al. 2002 - Whole rock and glass major-element geochemistry of Kilauea volcano, Hawaii, near-vent eruptive products : september 1994 through september 2001. USGS, Open file report p. 2-17.

RIVIÈRE E. 1894 - Nouvelles recherches anthropologiques et paléontologiques dans la Dordogne. Association française pour l'avancement des sciences, 23è session, Caen, p. 709-722.

\section{RÉSUMÉS}

Des polissoirs à rainures sont fréquemment décrits dans la littérature sur le Magdalénien. Le plus souvent, ils sont en grès mais quelques exemplaires sont en pierre ponce. La possible découverte d'un exemplaire de ce type dans le gisement de Duruthy (Sorde, Landes) nous a conduit à entreprendre une analyse texturale et géochimique du matériau. Les résultats indiquent qu'il s'agit d'une ponce rhyolitique. Un inventaire bibliographique fait ressortir que tous les objets similaires proviennent de sites préhistoriques de la moitié occidentale des Pyrénées. Ceci nous amène à suspecter qu'une éruption volcanique sur une île de l'océan Atlantique pourrait être à l'origine de ces ponces qui seraient arrivées sur les rivages par flottation avant d'y être collectées.

Magdalenian archaeological sites often contain grooves that were used to polish other implements (for example, Capitan and Peyrony 1928). These objects are generally made from sandstone but a few are made from pumice. A bibliographic search revealed that pumice stone polishers were found in only four sites (fig. 1, fig. 2, tabl. 1). They included: Gourdan (Piette 1873), Isturitz (Passemard 1944), Aitzbitarte IV (Barandiaran 1965) and Arancou (Chauchat et al. 1999). Several polishers were found in the rock shelter of Duruthy, Sorde-L'abbaye, Landes. Most of these polishers were sandstone (fig. 3) but one of them is different and has the appearance of pumice (fig. 4). The textural study and geochemical analysis (fig. 5, fig. 6, tabl. 2) of this polisher indicate that it was made from rhyolitic pumice. The geochemical characteristics of this material indicate that it is not a product of French volcanism. The fact that all the sites where pumice polishers were found are located nearby the Atlantic Ocean suggests that floated pumices may have been collected along the ocean shores. Such rhyolitic pumices could have been produced during the eruption of a volcano on an island in the Atlantic Ocean. Although we have not yet identified the volcano responsible of this eruption, it remains the most likely hypothesis. In this respect, these artefacts offer a new approach to the study of the exploitation of the littoral zone by the last palaeolithic hunters in southwest France and northen Spain. 
INDEX

Mots-clés : pierre ponce, polissoir à rainures, Pyrénées occidentales, Duruthy, Magdalénien, analyse géochimique, rhyolite

Keywords : Pumice, polisher with grooves, western Pyrenees, Duruthy, Magdalenian, geochemical analysis, rhyolite

\section{AUTEURS}

\section{MORGANE DACHARY}

UMR 5608 - TRACES. Université Toulouse-II-le Mirail. Maison de la recherche, 5 allée Antonio Machado, 31058 Toulouse cedex, France - morgane-dachary@orange.fr

\section{CATHERINE DENIEL}

Clermont Université, Université Blaise Pascal, Laboratoire Magmas et Volcans, BP 10448, 63000 Clermont-Ferrand - CNRS, UMR 6524, LMV et IRD, R 163, LMV, 63038 Clermont-Ferrand, France c.deniel@opgc.univ-bpclermont.fr

\section{FRÉDÉRIC PLASSARD}

UMR 5199, PACEA-Université Bordeaux 1, avenue des Facultés, 33400 Talence, France frederic.plassard@wanadoo.fr

\section{PIERRE BOIVIN}

Clermont Université, Université Blaise Pascal, Laboratoire Magmas et Volcans, BP 10448, 63000 Clermont-Ferrand - CNRS, UMR 6524, LMV et IRD, R 163, LMV, 63038 Clermont-Ferrand, France p.boivin@opgc.univ-bpclermont.fr

\section{JEAN-LUC DEVIDAL}

Clermont Université, Université Blaise Pascal, Laboratoire Magmas et Volcans, BP 10448, 63000 Clermont-Ferrand - CNRS, UMR 6524, LMV et IRD, R 163, LMV, 63038 Clermont-Ferrand, France j.l.devidal@opgc.univ-bpclermont.fr 